\title{
Comparing the association of cardiovascular nursing care with blood pressure and length of stay of in-patients with coronary artery disease in Wuhan, China
}

\author{
Fatina B Ramadhani ${ }^{1,2,3,4}$, Yilan Liu' ${ }^{1,2}$, Xue Jing ${ }^{1,3}$, Ye Qing ${ }^{1,3}$, \\ Han Xiong ${ }^{1}$, Fengiian Zhang ${ }^{1}$, Pian Wei ${ }^{5}$
}

1. Department of Nursing, Tongji Medical College, Huazhong University of Science and Technology

2. Department of Nursing, Union Hospital of Tongji Medical College, Huazhong University of Science and Technology

3. Department of cardiology, Union Hospital of Tongji Medical College, Huazhong University of science and Technology

4. Department of Clinical Nursing, Muhimbili University of Health and Allied Sciences

5. College of Arts and Science of Jianghan University

\begin{abstract}
Background: Coronary artery disease is a leading cause of morbidity and mortality worldwide. Comorbidity-like hypertension has been among the major risks of coronary artery disease. Recent evidence identified multiple benefits of cardiovascular nursing care to coronary patients. However, little has been appraised on benefits regarding patients' blood pressure control and length of hospitalisation.

Objective: To compare the association of cardiovascular nursing care delivered to coronary artery patients with patients' blood pressure and length of stay.

Methods: Records based retrospective design was applied at a large teaching hospital in Wuhan, China. SPSS 21 version was used for data entry and analysis with univariate and multivariate logistic regression models for comparing study variables.

Results: Of 300 patients, 224 (74.7\%) were known to be hypertensive and admitted with subnormal blood pressure. Cardiovascular nursing care like "assess to grade pain severity on 1-10 scale" and "counsel patient to cope with stress" were six and three times more likely to contribute improved patients' blood pressure (AOR=5.8; 95\%CI: 2.8-12.2, $\mathrm{p}=0.001$ ) and $(\mathrm{AOR}=3.1 ; 95 \% \mathrm{CI}: 1.2-7.8, \mathrm{p}=0.015)$ respectively. No significant difference with length of stay $(\mathrm{p}>0.05)$.

Conclusion: There is a possibility of coronary artery patients to recover with normal blood pressure following reception of evidence-based cardiovascular nursing care.

Keywords: Cardiovascular nursing care; blood pressure; in-patients; coronary artery; Wuhan; China.

DOI: https://dx.doi.org/10.4314/ahs.v20i4.23.

Cite as: Ramadhani FB, Lin Y, Jing X, Qing Y, Xiong H, Zhang F, et al. Comparing the association of cardiovascular nursing care with blood pressure and length of stay of in-patients with coronary artery disease in Wuban, China. Afri Health Sci. 2020;20(4):1716-24. https:// dx.doi.org/10.4314/ahs.v20i4.23
\end{abstract}

\section{Corresponding author:}

Fatina B Ramadhani,

Department of nursing, Tongji Medical

College, Huazhong Univeristy of Science

and Technology, 430030, Hubei, Wuhan, China.

Email: fatinarama27@yahoo.com

And

Yilan Liu,

Professor and director of nursing services,

Department of Nursing, Union

Hospital of Tongji Medical College,

Huazhong University of Science and Technology.

Email: yilanl2008@sina.com

\section{Introduction}

Coronary artery disease (CAD) is the principal global cause of morbidity and mortality that is responsible for more economic costs than any other single illness ${ }^{1,2}$. It is also the leading cause of death in China and increases economic health burden ${ }^{3}$. According to the 2015 China Cardiovascular Diseases (CVD) report, an epidemic of CVD is emerging as a result of lifestyle changes, urbanisation and the accelerated process of aging ${ }^{4}$. CAD is caused by atherosclerosis of the coronary arteries that leads to a restriction of blood flow to the heart 5,6. The worse form of CAD known as acute coronary syndrome (ACS) contributes to emergencies of hospital admissions ${ }^{2,7,8}$. Socio-demographic factors, such as old 
age, sex, family history of CAD and unhealthy lifestyles, obesity, smoking cigarettes and stress and comorbidities like hypertension, dyslipidaemia and diabetes mellitus have been universally reported as traditional risk factors of $\mathrm{CAD}^{9-11}$.

Approximately $75 \%$ of CAD can be attributable to conventional risk factors ${ }^{12}$. The modern treatment of CAD depends on the nature of coronary artery blockage or damage. These include vascular interventions such as percutaneous intervention (PCIs) (coronary stents and balloon angioplasty), coronary artery bypass graft (CABG), and pharmacological therapy ${ }^{13,14}$. Patients have also benefited and improved their quality of life by attending cardiac rehabilitation programmes ${ }^{15}$. As a result of this progress in treatment, the critical role of a nurse has also evolved.

\section{Nurses' roles and impact in caring for CAD patients}

For more than four decades, nurses have been taking key roles in managing single and multiple risk factors of CAD. This has been done through specialised clinics and programmes in primary care, worksites, and cardiac rehabilitation centres ${ }^{16,17}$. Provision of education on complex therapies in treating CAD, counselling on diet and lifestyle for risk factors modification and promotion on cardiac rehabilitation skills are among nursing roles delivered to patients with CAD ${ }^{14}$. A systematic review of randomised controlled trials revealed that nursing interventions (NI) for CAD patients have a beneficial impact on blood pressure (BP), lipids, physical activity, dietary intake, cigarette smoking, weight loss, healthcare utilisation, mortality, quality of life, and psychosocial outcomes. However, more than half of the interventions $(65 \%)$ were for education and behavioural counselling. Still inconsistent was the fact that many studies could not reveal which intervention feature was responsible for changes in outcome ${ }^{18}$.

Another review of randomised controlled trials conducted in homes and outpatient settings determined statistically significant impact of coordinated cardiovascular nursing care (CVNC) on the reduction of BP to CAD patients ${ }^{19}$.

The most recent systematic review and meta-analysis about the effective components of nurse-coordinated care to prevent recurrent coronary events also found heterogeneity of the data ${ }^{20}$. However, authors used descriptive approach to summarise NI components and their effect on outcomes. Basing on their consensus, they distinguished three intervention strategies: (1) risk factor management, for example, prescription and or titration of drug therapy, education on risk factors, counselling on diet and life style modification, vital signs monitoring like BP and lipid control and stress/ depressed mood detection and counselling;(2) multidisciplinary consultation, for example, consultation and referral and (3) shared decision making, for example, goal setting for individualised self-care plan and family support. Furthermore, important research questions were raised on how the proposed interventions can be translated into clinical practice, considering the fact that the statistically significant results may not represent clinically significant findings in all cases.

Basing on previous proposals, we published first the survey on the relevance of nursing caring interventions delivered to patients with CAD at a teaching hospital in Wuhan, China, by using patients' clinical records ${ }^{21}$. Results basically found eight cardiovascular nursing caring interventions related to the recommended evidence that were delivered to CAD patients. We also found a high rate of hypertensive patients 224/300 (74.7\%) with the history of about sixth readmission frequencies $165 / 300(55 \%)$. This part of the study aroused further synthesis of our results from previous published data which showed that most patients were admitted with subnormal blood pressure (BP). Some school of thought nicknamed hypertension (HT) as truly a "silent killer" due to its significant association of contributing to acute myocardial infarction (AMI) by 4.8 times among hypertensive as compared to normal subjects ${ }^{22}$. This implies a more indolent cardiac phenotype that if high BP is not well controlled would lead to more severe complications among CAD patients. Other studies suggested that preventive measures of HT at all levels should be given its special attention in minimising CAD episodes ${ }^{23}$.

Taking into consideration that high BP was earlier reported as among the major risks of CAD, we thought it was important to understand if CAD patients admitted with subnormal BP got any improvement following their hospitalisation. Therefore, the purpose of the current study was to investigate the hypothesis which stated that "CVNC has an association with the (improvement of coronary artery patients' blood pressure) and LOS". Publication of this study was expected to deliver further findings that appraise efforts of nurses in tackling CAD sequel to hospitalised patients and raise awareness on CAD illness to the readers of this article. 


\section{Methods}

\section{Study design and setting}

This survey was based on cross-sectional retrospective design. This study was conducted using patient's clinical records from November 10, 2017, to September 18,2018 , in sub-acute CAD wards at a specialised large teaching hospital in Wuhan, China ${ }^{21}$.

\section{Study participants and eligibility criteria}

The study targeted hospital registry of discharged cardiovascular patients who were having CAD diagnosis within the specified period of time. CV patient case note was reviewed if it met the following criteria: (1) had CAD diagnosis (2) had complete records (paper, electronic, or both) (3) was 18 years and above and sex demographic information (4) had at least one CVNC documentation (5) ward management permitted to use patients' records for this study. Excluded files were those of below November 10, 2017, or if just CVD related files, collapsed/died cardiovascular case and or due to other unforeseen reasons.

\section{Sample size and sampling}

A single population standard formula was used to calculate a sample size for this survey. This is a sample size calculated from a proportion of a single sampled study with similar or related characteristics of subjects for his study.

$\mathrm{n}=(\mathrm{Z}) 2 \mathrm{p}(1-\mathrm{p}) / \mathrm{d}^{2}$

$\mathrm{n}=$ Minimum sample size required for the study

$\mathrm{Z}=$ Value corresponding to the confidence level. For 0.05 confidence level $=1.96$

$\mathrm{p}=$ Prevalence of stable CAD in previous studies taken as $22.8 \%{ }^{21}$

$\mathrm{d}=$ Absolute precision (tolerable error) $=0.05$

$\mathrm{n}=(\mathrm{Z}) 2 \mathrm{p}(1-\mathrm{p}) / \mathrm{d}^{2}$

$\mathrm{n}=(1.96) 20.23(1-0.23) /(0.05)^{2}$

$\mathrm{n}=0.68=272.12 / 0.0025$

$\mathrm{n}=272.12+((10 \%) \mathrm{n})$ (note: $10 \%-15 \%$ are standard percentages normally added for increasing the sample size)

$\mathrm{n}=272.12+27.21=299.33$

Then approximately 300 files were selected by simple random technique from 700 eligible CAD patients files through excel sheet system.

\section{Instrument and data collection}

Data was collected by using a researcher-designed structured questionnaire. This approach was used due to the deficit of similar studies in the body of knowledge. Therefore, researchers designed the study questionnaire by adopting some categorical variables from previous related studies. However, "Any other (please specify)" option was purposively used to capture other information apart from those listed by the researchers in the questionnaire. The tool was divided into three sections. Section A: Composed of patient's demographic and clinical risk variables for CAD such as age, sex, smoking, and family history for CAD, hyperlipidaemia, diabetes mellitus and hypertension ${ }^{10,11,24}$. Section B: Composed of CVNC for CAD patients; such as education and counselling (18); administration of CAD medication and their instructions such as antiplatelet, promotion on diet, lifestyle and risk modification, selfcare and cardiac rehabilitation ${ }^{20,23,25,26}$. Part C: Composed of end clinical outcome variables such as length of stay (LOS) and discharge information of $\mathrm{BP}^{27,28}$. Thereafter, the designed questionnaire was translated to meet local language needs. A panel of experts in the fields of nursing research, management, education and cardiovascular assessed the content validity of the questionnaire. Pretest of the tool was done in 15 (5\%) questionnaires from clinical records of before November 2017. Also note that the pretesting did not include clinical records of died patients.

\section{Statistical analysis}

Data was analysed by using the Statistical Package for Social Sciences Software (SPSS) version 21. Continuous variables were calculated as mean \pm standard deviation (SD), and categorical variables were presented as counts (N) and percentages (\%). Univariate and multivariate logistic regression models were used to compare the relationship of independent (CVNC) and dependent variables (BP and LOS). P-value of less than 0.05 and adjusted odds ratios (AOR) with 95\% confidence interval (CI) within its ranges were stated as significant findings.

\section{Ethical considerations}

The study protocol was approved by research ethics committee of Tongji Medical College of Huazhong University of Science and Technology (IORG\#0003571). Furthermore, a verbal informed consent was obtained from hospitals' administrations to carry out the study and all patients details were kept anonymous.

\section{Results}

Demographic and CAD risk comorbid characteristics of patients. We audited a total of 300 CAD patients' 
clinical records. Most were males 175(58.3\%), their ages ranged from 33-99 with the mean age (63 111.2$)$ years (Table 1). Majority were married, 267 (89\%), while the rest were regarded as single and $278(92.7 \%)$ used health insurance to access health care services. Their levels of education and income generation activities also varied diversely. Majority 224 (74.7\%) were hypertensive. In these results, HT was among major modifiable CAD risk factors seen followed by diabetes mellitus 75 (25\%) and hyperlipidaemia 46(15.3\%). Other CAD risk life style behaviour like cigarette smoking was found to more than one third 126 (42.0\%). About 105 (35.7\%) of patients had CAD history in the family. Details of all demographics and most risky comorbidities of CAD patients are found in Table 1.

Table 1. Demographic and CAD risk comorbid characteristics of patients

\begin{tabular}{lll}
\hline Characteristics & & $\mathbf{n = 3 0 0}(\mathbf{\%})$ \\
\hline Gender & Male & $175(58.3)$ \\
Age & Female & $125(41.7)$ \\
& Below mean age $(63 \pm 11.2)$ & $150(50.0)$ \\
Education level & From mean age $(63 \pm 11.2)$ or above & $150(50.0)$ \\
& High school or below & $201(67.0)$ \\
Marital status & College or above & $99(33.0)$ \\
& asingle & $33(11.0)$ \\
Admission frequency & Married & $287(89.0)$ \\
& First & $135(45.0)$ \\
Method of payment & Second - sixth & $165(55.0)$ \\
& Health insurance based & $276(92.0)$ \\
Occupation & Cash based & $24(8.0)$ \\
& Employed & $73(24.3)$ \\
Smoking & Retired or self-employed or student & $227(75.7)$ \\
& Yes & $126(42.0)$ \\
CAD in family & No & $174(58.0)$ \\
& Yes & $107(35.7)$ \\
Hypertension & No & $193(64.3)$ \\
\multirow{2}{*}{ Hyperlipidaemia } & Yes & $224(74.7)$ \\
& No & $76(25.3)$ \\
Diabetes Mellitus & Yes & $46(15.3)$ \\
& No & $254(84.7)$ \\
& Yes & $75(25.0)$ \\
& No & $225(75.0)$ \\
\hline
\end{tabular}

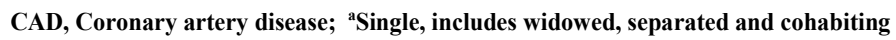

Comparing the association of CVNC with BP and LOS Tables 2 and 3 show statistical test results for comparing the association of cardiovascular nursing care with patients' blood pressure and length of stay. Note that documentation of this data was based on qualitative BP (whether normal or subnormal). Results show that less than quarter $40 / 300(13.3 \%)$ of patients were discharged with subnormal BP (Table 1). Patients spent an average of eight days ( $7.7 \pm 1.6$ days) of hospital admission. Categorical data analysis for LOS shows that about quarter $72 / 300$ (24\%) spent at least two weeks of hospitalisation (Table 2). 
Table 2. Comparing the association of CVNC with stability of patients' blood pressure

\begin{tabular}{|c|c|c|c|c|c|c|}
\hline \multirow{2}{*}{$\begin{array}{l}\text { Cardiovascular nursing care } \\
\text { delivered }\end{array}$} & \multicolumn{2}{|c|}{ CVNC $(n=300(\%))$} & \multicolumn{2}{|c|}{$\mathrm{BP}(\mathrm{n}=300)=\mathrm{N}(260): \mathrm{SN}(40)$} & \multirow[b]{2}{*}{$\mathrm{AOR}(95 \% \mathrm{CI})$} & \multirow[b]{2}{*}{$p$-value } \\
\hline & Found & Not found & Crude OR (95\%CI) & $p$-value & & \\
\hline $\begin{array}{l}\text { Assess to grade pain severity on 1-10 } \\
\text { scale }\end{array}$ & $206(68.7)$ & $94(31.3)$ & $2.4(1.1-5.6)$ & $0.048^{*}$ & $3.1(1.2-7.8)$ & $0.015^{*}$ \\
\hline Identify pain precipitating activities & $210(70.0)$ & $90(30)$ & $2.2(0.9-5.2)$ & 0.069 & $0.1(0.1-0.2)$ & 0.999 \\
\hline $\begin{array}{l}\text { Administer CAD medication and their } \\
\text { instructions }\end{array}$ & $290(96.7)$ & $10(3.3)$ & $1.4(0.2-11.3)$ & 0.754 & $0.1(0.1-0.2)$ & 1.000 \\
\hline $\begin{array}{l}\text { Educate on cardiac rehabilitation } \\
\text { skills, exercise }\end{array}$ & $285(95.0)$ & $15(5.0)$ & $1.0(0.2-4.6)$ & 1.000 & $1.5(0.1-17.9)$ & 0.760 \\
\hline $\begin{array}{l}\text { Counsel on diet and life style } \\
\text { modification }\end{array}$ & $289(96.3)$ & $11(3.7)$ & $1.6(0.2-12.5)$ & 0.676 & $4.7(0.4-5.6)$ & 0.219 \\
\hline $\begin{array}{l}\text { Offer reassurance and family } \\
\text { counselling }\end{array}$ & $284(94.7)$ & $16(5.3)$ & $0.4(0.1-1.4)$ & 0.169 & $0.6(0.1-5.0)$ & 0.678 \\
\hline Monitor vital signs & $235(78.3)$ & $65(21.7)$ & $1.4(0.6-3.2)$ & 0.493 & $1.3(0.4-3.7)$ & 0.646 \\
\hline Counsel to cope with stress & $60(20.0)$ & $240(80.0)$ & $4.8(2.4-9.8)$ & $0.001 * *$ & $5.8(2.8-12.2)$ & $0.001^{* *}$ \\
\hline
\end{tabular}

CAD, Coronary artery disease; CVNC, Cardiovascular nursing care; BP, Blood pressure; N, Norma; SN, Sub-normal; OR, Odds Ratio; AOR, Adjusted Odds Ratio; CI, Confidence Interval; *p $<0.05 ; * * p<0.001$

Table 3. Comparing the association of CVNC with patients' length of hospital stay

\begin{tabular}{lllll}
\hline Cardiovascular nursing care & \multicolumn{2}{c}{ CVNC (n=300 (\%)) } & \multicolumn{1}{c}{ LOS(n=300)= 8D=228: 9-15D=72 } \\
\cline { 2 - 5 } & Found & Not found & Crude OR (95\%CI) & $p$-value \\
\hline Assess to grade pain severity on 1-10 scale & $206(68.7)$ & $94(31.3)$ & $1.1(0.6-1.9)$ & 0.870 \\
Identify pain precipitating activities & $210(70.0)$ & $90(30)$ & $1.3(0.7-2.3)$ & 0.444 \\
Administer CAD medication and their instructions & $290(96.7)$ & $10(3.3)$ & $0.7(0.2-2.9)$ & 0.653 \\
Educate on cardiac rehabilitation skills, exercise & $285(95.0)$ & $15(5.0)$ & $0.4(0.2-1.3)$ & 0.145 \\
Counsel on diet and life style modification & $289(96.3)$ & $11(3.7)$ & $0.8(0.2-3.2)$ & 0.796 \\
Offer reassurance and family counselling & $284(94.7)$ & $16(5.3)$ & $0.9(0.3-3.0)$ & 0.923 \\
Monitor vital signs & $235(78.3)$ & $65(21.7)$ & $0.9(0.5-1.8)$ & 0.896 \\
Counsel to cope with stress & $60(20.0)$ & $240(80.0)$ & $0.8(0.4-1.7)$ & 0.636 \\
\hline
\end{tabular}

CAD, Coronary artery disease; CVNC, Cardiovascular nursing care; LOS, Length of stay; D, Days; OR, Odds Ratio; CI, Confidence Interval

Both univariate and multivariate logistic regression analysis were run in SPSS 21 version to determine this association. In this analysis, CVNC was an independent variable on impacting patients' BP and LOS as dependent variables. After adjusting for patient's demographics, clinical characteristics and readmission information results show that CVNC, like "counsel patient to cope with stress" and "assess to grade pain severity on 1-10 scale," were six and three times more likely to contribute to improved BP of patients following their hospitalisation (AOR $=5.8$; 95\%CI: 2.8-12.2, $\mathrm{p}=0.001)$ and $(\mathrm{AOR}=3.1 ; 95 \% \mathrm{CI}: 1.2-7.8, \mathrm{p}=0.015)$ respectively (Table 2). However, no significant difference found with LOS ( $p>0.05)$ (Table 3). 


\section{Discussion}

The current study compared the association of cardiovascular nursing care (CVNC) with BP and LOS of hospitalised CAD patients at a large specialised teaching hospital in Wuhan, China. Mean age of patients was $63 \pm 11.2$ years. This reflects older adults due to the fact that CAD is historically known as a disease of elderly as it develops gradually with aging. However, we observed half of the study population was younger than 60 years. This means CAD is currently affecting younger adults too. Our interpretation is similar to the finding which reported that CAD still affects majority of old adults with an upcoming trend of middle to younger adults 15,21 .

Consistently, the disease may become clinically apparent by age 40 , but prevalence is higher in people 65 years of age and older ${ }^{29}$.

From this study, three quarters of patients were hypertensive. This observation is similar to the findings where by hypertension had the leading proportion $(80.7 \%)$ of all chronic comorbidity which were significantly associated with CAD in hospitalised cardiovascular patients ${ }^{30}$. In China, the prevalence of hypertension among subjects aged 15 years and above were reported to increase and also increase the risks of CVD and health expenditure. The major risk factors were high sodium and low potassium diet, obesity, overweight, high alcohol consumption, mental stress, family history of hypertension, and sedentary lifestyle ${ }^{4}$. Several studies have listed hypertension as one of the leading comorbidities among CAD patients ${ }^{31}$.

Our principle findings reveal significant association between CVNC "counsel patient to cope with stress" and "assess to grade pain severity on 1-10 scale" and BP. Where their relationship had positive beta values, hence indicates significant contribution of some nursing procedures in controlling patients from subnormal on admission to normal during discharge. The current study indicates that pain management has been one of the major focuses of nurses to CAD patients that had positive significant impact on BP. Nursing pain care is implemented as a response to a nursing diagnosis that is related by the bio physiologic injury secondary to decreased oxygen supply to the myocardium ${ }^{32}$. Pain interventions offered go online and reflect the need of CAD patients whom cardiac chest pain is usually their major symptom. This finding is reflected in the results from a systematic review whereby nurse-led clinic group showed a significant decrease of worsening chest pain among patients who experienced angina as compared to those on usual care ${ }^{19}$. Relieving chest pain would probably reflect improvement of coronary vascular blood flow, hence reduction of BP from vascular constriction.

However, fewer patients (20\%) of 300 received nursing care on copying with stress.

Psychological factors such as depression and stress have been listed as among the underlying risk factors for $\mathrm{CAD}^{33}$. Individuals who adjust CAD risk factors in a constructive direction can lower the risk for CAD ${ }^{34}$. Regardless of the fact that nurses can face time constraints and inadequate staffing, nursing practices can still be developed with technology that improves the physical environment to provide CAD patients with the opportunity to feel better about themselves ${ }^{35}$. Therefore, we recommend increased efforts in managing patients' stress to minimise triggering CAD attacks for promoting patients' wellbeing.

This study faced difficulties of tracing some surgical and pharmacological records. The collaborative management was not adjusted for this study, hence should be of future focus to properly appraise the pure nursing efforts of relieving CAD patients' BP. Other studies revealed that multidisciplinary secondary prevention have a beneficial effect on process of care in patients with $\mathrm{CAD}^{27}$. Therefore, future surveys can be done in similar or multiple settings to challenge these findings. Our findings are somehow consistent with other reports which revealed that the decline in-hospital acute CAD conditions like myocardial infarction (AMI) reflects advances in treatment and nurses efforts in timelines administration and monitoring side effects of reperfusion therapy ${ }^{25,26,36}$.

Moreover, the current findings show that patients spent

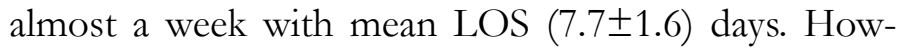
ever, the current study has no enough evidence on the relationship of CVNC with LOS. Other findings reported that $\mathrm{CAD}$ patients with comorbidity conditions significantly spent more days of hospitalisation than those who had no chronic conditions ${ }^{30}$. CAD patients were said to form a non-uniform group and their prognosis may differ depending on demographic, clinical, geographic, and socioeconomic factors ${ }^{37}$. Therefore, basing on such findings, this relationship could probably be well explained by clinical characteristics or other factors.

Furthermore, we found more than half $(55 \%)$ of 300 patients experienced and increased trend of readmissions from two to six times. This was also reported in 
studies where patients with CAD conditions like myocardial infarction (MI) faced some hospital readmis$\operatorname{sion}^{27}$.

Readmission frequencies could be associated with knowledge deficit in controlling their illness.

Studies explored that through knowledge assessment, patient education, discharge preparation, and care-coordination; nurses can be the frontline for providing many of the core processes of care aimed at preventing readmissions ${ }^{38}$. Therefore, this study calls for a need of action in improving patients' knowledge of an illness and their well-being, hence minimise hospitalisation and related economic burdens to themselves, their families and nation at large.

To the best of our knowledge, availability of similar kind of study was limited, hence we faced deficit of enough comparable findings. However, benefits of CVNC that were found in the systematic reviews of randomised controlled trials have reflected current results ${ }^{39}$. However, these kinds of studies are still inadequately conducted in the clinical settings. Hence further researches are needed to confirm its clinical significance.

Limitations of our survey include; being a retrospective of clinical patient's registry where some data was not complete, hence we couldn't get all numerical BP measurements of actual patients. Because of this, we opted for qualitative BP data (whether recorded as normal or subnormal), hence we were not able to measure differences of BP readings during hospital admission and discharge. This also limited the interview of patients who agreed to be discharged with unstable blood BP findings.

Additionally, there were insufficient surgical and pharmacological records as none nursing interventions to adjust in the statistical analysis of this association. Therefore, our study might have limited generalisability, hence could be appropriate for CVNC delivered to the population attending in the teaching hospital under this study.

\section{Conclusion}

This study reveals that CAD is currently not only affecting older adults but also people below 60 years of age. Modifiable risk factors like hypertension are still increasing to compromise the health of CAD patients. Cardiovascular nursing care such as "counsel patient to cope with stress" and "assess to grade pain severity on 1-10 scale" significantly contributed to the correction of subnormal BP to normal of hospitalised CAD patients. However, this conclusion could be less solid due to small number of patients who received " help patient cope with stress" as well as insufficient comparison with other none nursing interventions. Hence further study is needed to fill these gaps and to properly appraise typical nursing efforts of caring CAD patients. Moreover, assessment of patients' knowledge on components on cardiac rehabilitation and related ill health behaviours is paramount in improving their health, minimising readmission frequencies and economic burden from CAD.

\section{Conflict of interest}

None declared.

\section{References}

1. World Health Organization. Cardiovascular diseases, fact sheet \#317. 2015. Available from: http://www. who.int/mediacentre/factsheets/fs317/en/

2. Singh V, Kumari G, Chhajer B, Dahiya S, Rumaneh W, Mazzaferro E, et al. Epidemiology of coronary heart disease and acute coronary syndrome. Ann Transl Med. 2017;4(1):256-256. Available from: http://www. mountsinai.org/interventional-cardiology-cath-lab/ conditions-we-treat/coronary-artery-disease

3. Li H, Ge J. Cardiovascular diseases in China: Current status and future perspectives. IJC Hear Vasc. 2015;6:2531. Available from: http://dx.doi.org/10.1016/j.ijcha.2014.10.002

4. Chen W-W, Gao R-L, Liu L-S, Zhu M-L, Wang W, Wang Y-J, et al. China cardiovascular diseases report 2015: a summary. J Geriatr Cardiol. 2017;14(1):1-10. Available from: http://www.ncbi.nlm.nih.gov/pubmed/28270835\%0Ahttp://www.pubmedcentral.nih. gov $/$ articlerender.fcgi?artid=PMC5329726

5. Katz MJ, Ness SM. Coronary Artery Disease (CAD). Iris, Wild Educ Med. 2015;1-12. Available from: http://www.mountsinai.org/interventional-cardiology-cath-lab/conditions-we-treat/coronary-artery-disease

6. Sanchis-Gomar F, Perez-Quilis C, Leischik R, Lucia A. Epidemiology of coronary heart disease and acute coronary syndrome. Ann Transl Med. 2016;4(13):256256. Available from: http://atm.amegroups.com/article/view/10896/11530

7. Bhagwat MM, Woods JA, Dronavalli M, Hamilton SJ, Thompson SC. Evidence-based interventions in primary care following acute coronary syndrome in Australia and New Zealand: a systematic scoping review. BMC Cardiovasc Disord. 2016; Available from: http://dx.doi. org/10.1186/s12872-016-0388-y

8. Andrikopoulos G, Terentes-Printzios D, Tzeis S, Vlachopoulos C, Varounis C, Nikas N, et al. Epidemio- 
logical characteristics, management and early outcomes of acute coronary syndromes in Greece: The Phaenthon study. Hell J Cardiol. 2016;57(3):157-66.

9. Smith SCJ, Benjamin EJ, Bonow, R.O, Braun LT, Creager, M. A, Franklin BA, Gibbons RJ, Grundy, $\mathrm{S} \mathrm{M}$, et al. AHA/ACCF secondary preven- tion and risk reduction therapy for patients with coronary and other atherosclerotic vascular disease: 2011 update: a guideline from the American heart association and American college of cardiology foundation. Circulation. 2011;124(1):2458-2473.

10. Mohammad AM, Jehangeer HI, Shaikhow SK. Prevalence and risk factors of premature coronary artery disease in patients undergoing coronary angiography in. BMC Cardiovasc Disord. 2015;1-6. Available from: http://dx.doi.org/10.1186/s12872-015-0145-7

11. Krishnan MN, Zachariah G, Venugopal K, Mohanan PP, Harikrishnan S, Sanjay G, et al. Prevalence of coronary artery disease and its risk factors in Kerala, South India : a community-based cross-sectional study. BMC Cardiovasc Disord. 2016; Available from: http://dx. doi.org/10.1186/s12872-016-0189-3

12. Mackay J, Mensah GA. Global burden of coronary heart disease Healthy years of life lost to coronary heart disease. The Atlas of Heart Disease and Stroke. 2004. p. 46-7. Available from: https://www.who.int/ cardiovascular_diseases/en/cvd_atlas_03_risk_factors. pdf?ua $=1$

13. Honeycutt E, Roe MT. Percutaneous Coronary Intervention in Native Arteries Versus Bypass Grafts in Prior Coronary Artery Bypass Grafting Patients. J Cardiovasc Interv. 2011;4(8):844-50. Available from: http:// dx.doi.org/10.1016/j.jcin.2011.03.018

14. Berra K, Fletcher, J. B, Handberg E. Antiplatelet Therapy in Acute Coronary Syndromes Implications for Nursing Practice. J Cardiovasc Nurs. 2011;26(3):23949.

15. Piepoli MF, Hoes AW, Agewall S, Albus C, Brotons C, Catapano AL, et al. 2016 European Guidelines on cardiovascular disease prevention in clinical practice. Eur Heart J. 2016;37(29):2315-81.

16. Wal P, Wal A, Rai A, Nair V, Pandey U. Management of coronary artery disease in a Tertiary Care Hospital. J Basic Clin Pharm. 2013;4(2):31. Available from: http:/ / www.jbclinpharm.org/text.asp?2013/4/2/31/113605 17. Yang Q, Wang Y, Liu J, Liu J, Hao Y, Smith SC, et al. Invasive Management Strategies and Antithrombotic Treatments in Patients With Non - ST-Segment - Elevation Acute Coronary Syndrome in China. Circ Cardiovasc Interv. 2017;
18. Allen JK, Dennison CR. Randomized Trials of Nursing Interventions for Secondary Prevention in Patients With Coronary Artery Disease and Heart Failure Systematic Review. J Cardiovasc Nurs. 2010;25(3):207-20. 19. Health Quality Ontario. Specialized nursing practice for chronic disease management in the primary care setting: an evidence-based analysis. Ont Health Technol Assess Ser. 2013;13(10):1-66.

20. Snaterse M, Dobber J, Jepma P, Peters RJG, Riet G Ter, Boekholdt SM, et al. Effective components of nurse-coordinated care to prevent recurrent coronary events: A systematic review and meta-analysis. Heart. 2016;102(1):50-6.

21. Ramadhani FB, Jing X, Qing Y, Rathnayake AK, Kara WSK, Wu W, et al. Investigating the Relevance of Nursing Caring Interventions Delivered to Patients with Coronary Artery Disease at a Teaching Hospital in China: A Retrospective Study. Cureus. 2019;11(5).

22. Ades PA. Secondary Prevention of Coronary Heart Disease in Patients With Coronary. $N$ Engl J Med. 2004;345(12):892-902.

23. Leon AS, Franklin BA, Costa F, Balady GJ, Berra $\mathrm{KA}$, Stewart KJ, et al. Cardiac rehabilitation and secondary prevention of coronary heart disease: An American Heart Assoc.scientific statement from the Council on Clin. Cardiol. (Subcommittee on Exercise, Cardiac Rehabil., and Prevention) and the Council on Nutr., Phys. Activi. Circulation. 2005;111(3):369-76.

24. Khalili D, Sheikholeslami FH, Bakhtiyari M, Azizi F. The Incidence of Coronary Heart Disease and the Population Attributable Fraction of Its Risk Factors in Tehran : A 10-Year Population-Based Cohort Study. PLoS One. 2014;9(8):1-7.

25. Hayman LL, Berra K, Fletcher BJ, Houston Miller $\mathrm{N}$. The role of nurses in promoting cardiovascular health worldwide: The global cardiovascular nursing leadership forum. J Am Coll Cardiol. 2015;66(7):864-6. 26. Berra K, Fletcher BJ, Hayman LL, Miller NH. Global cardiovascular disease prevention: A call to action for nursing The global burden of cardiovascular disease. Eur J Cardiovasc Nurs. 2011;10(SUPPL. 2).

27. Meisinger C, Stollenwerk B, Kirchberger I, Seidl $\mathrm{H}$, Wende R, Kuch B, et al. Effects of a nurse-based case management compared to usual care among aged patients with myocardial infarction: Results from the randomized controlled KORINNA study. BMC Geriatr. 2013;13(1):1. Available from: BMC Geriatrics

28. Stylianou N, Fackrell R, Vasilakis C. Are medical outliers associated with worse patient outcomes? A retrospective study within a regional NHS hospital using routine data. 2017; 
29. Kendir C, van den Akker M, Vos R, Metsemakers J. Cardiovascular disease patients have increased risk for comorbidity: A cross-sectional study in the Netherlands. Eur J Gen Pract. 2018;24(1):45-50. Available from: https://doi.org/10.1080/13814788.2017.1398318

30. K Murray M-I, Thalmann IN, Mossialos EA, Zeiher AM. Comorbidities of Coronary Heart Disease and the Impact on Healthcare Usage and Productivity Loss in a Nationally-Based Study. Epidemiol Open Access. 2018;08(03).

31. Köhler AK, Tingström P, Jaarsma T, Nilsson S. Patient empowerment and general self-efficacy in patients with coronary heart disease: a cross-sectional study. 2018;1-10.

32. Swearingen MBJKP. Manual of Critical Care Nursing: Nursing Interventions and Collaborative Management. $6^{\text {th }}$ ed. St. Louis Missouri: Mosby Elsevier; 2011. 33. Kazemian F, Jalali SF, Hajian-Tilaki K, Arzani A AKR. Underlying risk factors and their relationship with extent of coronary vessel involvement in patients undergoing coronary angiography in North of Iran. 2018;9(April):361-7.

34. Ahmad M, Tawalbeh L. Patients with Coronary Artery Disease in the North of Jordan Toward Healthy Lifestyle Intervention. Int J Public Heal Res. 2015;3(5):279-87.
35. Nural N, Alkan S. Identifying the Factors Affecting Comfort and the Comfort Levels of Patients Hospitalized in the Coronary Care Unit. Holist Nurs Pract. 2018;32(1):35-42.

36. Staniūtè M, Brožaitienè J. Changes in health-related quality of life among patients with coronary artery disease: a 2-year follow-up. Medicina (Kaunas). 2010;46(12):843-50. Available from:http://www.ncbi. nlm.nih.gov/pubmed/21532289

37. Parma Z, Steg PG, Greenlaw N, Ferrari R, Ford I, Fox K, et al. Differences in outcomes in patients with stable coronary artery disease managed by cardiologists versus noncardiologists. Results from the international prospective CLARIFY registry. Polish Arch Intern Med. 2017;127(2):107-14.

38. McHugh MD, Ma C. Hospital nursing and 30-day readmissions among Medicare patients with heart failure, acute myocardial infarction, and pneumonia. Med Care. 2013;51(1):52-9.

39. Wong N, Chua SJT, Gao F, Sim STR, Matchar D, Wong SLA, et al. The effect of a nurse-led telephone-based care coordination program on the follow-up and control of cardiovascular risk factors in patients with coronary artery disease. Int J Qual Heal Care. 2016;28(6):758-63. 